\title{
Quantitative detection of MMP-2 and relationship of the clinical features and prognosis of lung cancer
}

\author{
Jie MA \\ Langfang Health Vocational College \\ Hebei,065001 China \\ Haiping PENG \\ Langfang Health Vocational College \\ Hebei,065001 China
}

\author{
Haiyuan PENG \\ Langfang People's Hospital \\ Hebei,065000 China \\ Huifang ZHU \\ Langfang Health Vocational College \\ Hebei,065001 China
}

\begin{abstract}
Objective is to discuss (MMP-2) relationship of matrix metalloproteinase-2 and lung cancer invasion, metastasis and prognosis. Methods: use flow immunoassay for the quantitative detection of MMP-2 expression in 28 cases of benign lung lesions and 78 cases of lung cancer. Results: MMP 2-expression promotes lung cancer is higher than benign lesions ( $p<0.05)$, and there is a significant correlation $(p<0.05)$ of lung cancer clinical stage and histological grade. Conclusion: The high expression of MMP-2 promotes the invasion and metastasis of lung cancer is, the higher the value is, the higher the clinical stage is, and the lower the tissue differentiation is, suggesting that MMP-2 not only can be used as indicators of lung cancer, but also is effective prognosis.
\end{abstract}

Keywords- lung cancer, MMP-2, gene expression, invasion and metastasis, flow cytometry.

\section{INTRODUCTION}

Lung cancer incidence and mortality rate ranks first in all kinds of malignant tumors, and tumor invasion and metastasis is the main cause of death in patients. [1] Recent studies have shown that tumor invasion and metastasis is a complex, multi-factor involved process, it includes the loss from the primary site, extracellular matrix degradation breaking through the basement membrane into the circulatory system, migration, adhesion, and colonization and so on. Matrix metalloproteinase -2 (MMP-2) is the degradation of extracellular matrix and basement membrane of the main enzymes, thereby promoting tumor invasion and metastasis. In this study, through flow cytometry (FCM) quantitative detection of MMP-2 expression in lung tissue, we explore lung cancer's occurrence, development, invasion and metastasis role in lung cancer to evaluate the degree of malignancy and clinical prognosis in value.

\section{SUBJECT AND MethodS}

\section{A. Object}

Select lung tissue archival paraffin blocks 106 as cases, paraffin blocks are selected from 2006 to 2013 archival paraffin Langfang City People's Hospital. 28 cases are benign lung lesions, and 78 cases are lung cancer. 78 cases of lung cancer are ranked according to TNM staging criteria; I have 10 cases, II have 14 cases, III have 15 cases, IV has 39 cases. 22 cases are squamous cell carcinoma, adenocarcinoma has 28 cases, 6 cases are large cell carcinoma and glandular squamous cell carcinoma has 10 cases, 12 cases are small cell carcinoma differentiation, 78 cases according to histological grading of Class have I, II grade (high grade) 35 cases, grade III (poorly) 43 cases. The average age Selection of cases is 47.5 years. We also select 10 cases of normal lung tissue as control.

\section{B. Methods:}

Use Epics-XLII flow cytometer and the organization wax block flow immunoassay for the quantitative detection of the expression levels of MMP-2. Wax block single cell suspension was prepared by conventional treatment, use indirect immunofluorescence labeling of MMP-2, flow cytometry, laser excitation source is $15 \mathrm{~mW}$ argon ion, and a wavelength is of $488 \mathrm{~nm}$, use Expo32ADC immunofluorescence data analysis. In addition to the control group of normal lung tissue, with a reagent antibody (mouse anti-human MMP-2 monoclonal antibody) secondary antibody (goat anti-mouse FITC-IgG) tissue markers are set in the control group. Ultimately calculate the fluorescence index (Fluorescence Idex, FI) = mean fluorescence intensity of the sample protein expression (mode value) / mean fluorescence intensity of the control samples (mode value) to FI represents the relative content of protein in the tissue expression of the gene. Use SPSS17.0 statistical software, $t$ test, analysis of variance, data analysis, rank correlation analysis, when $\mathrm{P} \leqslant 0.05$, there are statistical significance. 


\section{RESULTS}

\section{A. MMP-2 gene expression in lung tissue protein (Table 1)}

MMP-2 protein expression in lung volume average FI is $2.98 \pm 1.04$, which is significantly higher than in normal lung tissue $(\mathrm{FI}=1.00 \pm 0.23)$ and pulmonary benign lesions $(\mathrm{FI}=$ $1.54 \pm 0.31)$, the differences were statistically significant $(\mathrm{p}$ $<0.05)$.

Table 1 MMP-2 gene expression in lung tissue protein

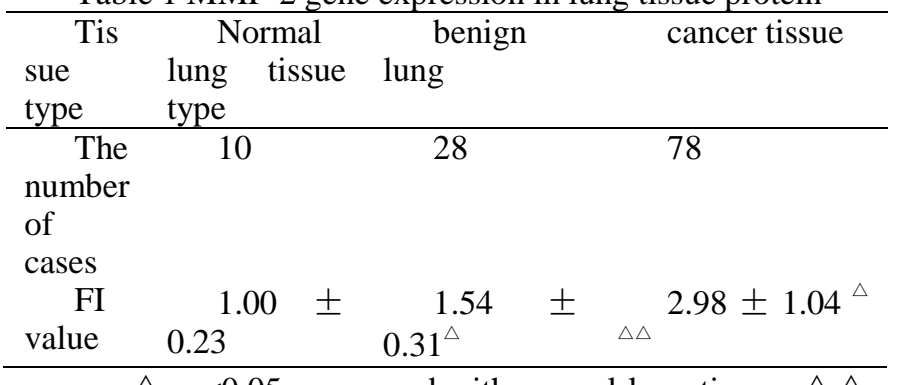

$\triangle \mathrm{p}<0.05$, compared with normal lung tissue; $\triangle \triangle$

$\mathrm{p}<0.05$, compared with benign lung disease;

\section{B. MMP-2 gene expression of proteins in lung cancer}

Class III (poorly differentiated) cancer MMP-2 protein expression FI average value is $3.55 \pm 0.86$, which is significantly higher than I, II level (high grade) lung cancer, and the differences were statistically significant ( $\mathrm{p}<0.05)$. III, IV stage lung cancer MMP-2 protein expression mean FI is $3.52 \pm 1.04$, which is significantly higher than I, II lung cancer, and the differences were statistically significant $(\mathrm{p}$ $<0.05)$.

Table 2 MMP-2 gene expression of proteins in lung cancer

\begin{tabular}{|c|c|c|c|c|}
\hline \multirow[b]{2}{*}{$\begin{array}{l}\text { Lung } \\
\text { cancer }\end{array}$} & \multicolumn{2}{|c|}{ Tissue classification } & \multicolumn{2}{|c|}{ clinical staging classification } \\
\hline & $\begin{array}{l}\text { Ilevel II } \\
\text { level ( (high } \\
\text { grade) }\end{array}$ & $\begin{array}{l}\text { IIIlevel } \\
\text { (low grade) }\end{array}$ & I、 IIperiod & III IVperiod \\
\hline $\begin{array}{l}\text { Case } \\
\text { number }\end{array}$ & 35 & 43 & 24 & 54 \\
\hline $\begin{array}{c}\text { FI } \\
\text { value }\end{array}$ & $\int_{0.78}^{2.42} \pm$ & $3.55 \pm 0.86^{\boldsymbol{\Delta}}$ & $2.41 \pm 0.81$ & $3.52 \pm 1.04 *$ \\
\hline
\end{tabular}

$\Delta \mathrm{p}<0.05$, with I, II stage lung cancer than; $* \mathrm{p}<0.05$, with I, II lung cancer ratio.

\section{DISCUSSIONS}

Lung cancer has become the most common malignant tumors in recent years, the incidence and number of lung cancer deaths is to rise. Lung cancer incidence and mortality are at the head of malignant tumors. [2] Although the past half century, the comprehensive treatment of lung cancer has made important progress, but the 5-year survival rate is very low. The main reason is the inadequate procedures for routine screening of lung cancer, the cancer is not recognized early damage, and early diagnosis rate is $15 \%$. Many tumor cell metastasis and spread of lung cancer prognostic factors are major cause of death. Reduce the tumor cell adhesion, basal secretions of extracellular matrix degrading enzymes degrade the invasion and metastasis. These are related to gene expression of MMP-2.

Invasion and metastasis of lung cancer is a multi-factor, multi-step, complex multi-stage process, including invasion, proliferation cycle, distant cloning and angiogenesis. Extracellular matrix degradation is a key step in which, while MMP-2 can degrade the vascular basement membrane and extracellular matrix components collagen IV, completed extracellular matrix degradation and the tumor cells are easily break through the barrier structure invasion or into the blood circulation or lymphatic circulation, or into the distant tissues and organs implantation, the surrounding tissue forms metastases. Thus, MMP-2 is considered to be malignant cell invasion and metastasis potential signs. Studies have shown that MMP-2 plays a very important role in cancer cells falling off, spreading, invasion and metastasis.

Sun Shuming[3] uses immunohistochemistry in 71 cases of lung cancer specimens and 31 cases of normal lung tissue to detect the expression of MMP-2 studies, studies show that MMP-2 positive cancer cells were spotty distribution, and more are in the cancer edge Tip and more cancer cells have a peripheral surface or located tumor invasion potential. Xue Yang [4] uses immunohistochemistry for the expression levels of 108 cases of non-small cell lung cancer and 19 patients with benign lung lesions were detected MMP-2, studies have shown that lung levels of MMP-2 expression was significantly increased, and Lung tissue levels of MMP2 expression was significantly positively correlated; progression and metastasis is proportional in lung cancer. Pan Qideng [5] uses quantum dot immunofluorescence techniques to detect the expression 70 cases of lung cancer and 10 cases of lung cancer in non-MMP-2 and MMP-2 
study shows that in the non-cancerous lung tissue has almost no expression in lung cancer MMP- the positive rate compared with control tissue two significant differences, indicating the occurrence of lung expression of MMP-2. The positive rate of MMP-2 and high TNM stage and lymph node metastasis was significantly positively correlated. The results of the study show lung MMP-2 protein expression (FI value) increases, and it is significantly higher than in normal lung tissues and benign lesions, suggesting that MMP-2 is related to the development of lung cancer. This is pertain to Sun Shuming etc., Xue Yang and other findings.

Peng Liang [6] detected radioimmunoassay of serum MMP-2 expression in 80 patients with small cell lung cancer, and studies have shown that patients with lung cancer was significantly higher than the control group, and extensive stage patients was significantly higher in patients with limited, suggesting MMP- 2 expression and the development of small-cell lung cancer have an important role in the invasion and metastasis and is closely related to the degree of malignancy. The results of the study show III, IV lung cancer MMP-2 protein expression was significantly higher than the average FI stage II and stage I lung cancer, indicating that MMP-2 protein expression is closely related to the clinical stage ( $\mathrm{p}<0.05)$. This is basically consistent with the findings of Xue Yang et al., Panqi Deng, Peng Liang.

Oman Wang et al [7] use immunohistochemical method to detect 42 cases of small cell carcinoma of the MMP-2 expression and its relationship with pathological type and prognosis, MMP-2 was found involved in the invasion and metastasis of lung cancer, and it can predict to a certain extent prognosis. Wang Libo, etc. [8] use immunohistochemistry to detect 62 cases of non-small cell lung cancer and found no correlation between the expression of MMP-2 and tissue type and tumor size, positive rate increased with clinical stage and histological grade level increased lymph node metastasis, the tumors were significantly higher than those without lymph node metastasis, suggesting that MMP-2 expression and metastasis of non-small cell lung cancer are related. Passlick and other prospective study have concluded that, MMP-2 overexpression is poor prognosis in early stage NSCLC. This study showed grade III (poorly) in lung cancer MMP-2 protein expression was significantly higher than the average FI grade I, II grade (high grade) of lung cancer, suggesting that MMP-2 and tissue-related lung cancer classification. This is similar to Passlick etc. Oman Wang, Wang Libo, etc.,Peng Liang findings.

This study shows that the quantitative detection of MMP2 protein FI value and it can reflect the MMP-2 in the development of lung cancer, and it plays an important role in the invasion and metastasis of tumors. MMP-2 protein expression is closely related to the degree of malignancy, clinical stage and the extent of lung tissue differentiation; prognosis can be a certain extent.

\section{CONCLUSIONS:}

In summary, this study showed that MMP-2 is closely related to lung tumor invasion and metastasis, and its expression in lung cancer detection is in order to further expand the number of samples to investigate the clinical and pathological diagnosis of the molecular mechanisms of lung cancer development for lung cancer treatment and prognosis laid the foundation for the diagnosis and treatment of lung cancer and provides an important target. Lung cancer prognosis, lung cancer prognostic indicators are to explore, to objectively evaluate the clinical effect, to further improve the comprehensive treatment programs to improve the efficacy, and it has clinical significance.

\section{ACKNOWLEDGEMENTS}

2014 Langfang Municipal Science and Technology Research and Development Program: MMP-9, MMP-2 and E-cadherin and lung cancer clinical features and prognosis of clinical studies, the number 2014013033, first Zhuyanren, Peng seafarers

\section{References}

[1] Spiro SG, Silvestri GA One hundred years of lung cancer [J] Am J Respir Crit Care Med, 2005, 172 (5): 523-529.

[2] Daimin, Renjian Song, Li Ni China 2008 and other cancer incidence and mortality estimates and forecast $[\mathrm{J}]$ Chinese Journal of Epidemiology, 2012; 33 (1): 57-61.

[3] Sun Shuming, Zhou Yan, Luo Jinfang, Gui Lv, Hu Zhixiong, Jin Ying, Jin Fusheng MMP-2 and MMP-9 expression in lung cancer [J] Journal of Cancer Prevention .2003,10 (9): 920-922.

[4] Xue Yang, Zhou Qinghua, Zhang Shangfu, Liu LunXu. MMP-2, MMP-9 expression and its relationship with lung metastasis and prognosis in lung cancer.[J]. West China Medical .2008,23 (2), 225 227.

[5] Pan Qi, Fuchun Chen, Wu Baiqiang, Bao Jun, Xue Jiehao, Chen Honglei quantum dots immunofluorescence EMMPRIN, expression meaning. MMP-2 and P53 protein in human lung cancer[J]. Chinese Journal of Pathophysiology .2011, 27 (3): 488-493.

[6] Peng Liang .MMP-2 and TIMP-2 in the serum of patients with small cell lung cancer and its clinical significance [J] RIA Journal .2008,21 (6): 523-524

[7] Wang Aman, CAI Xin, Zhou Tao, Liu Ji Wei .MMP-2, VEGF, CD105 and Ki-67 expression and prognosis studies in small cell lung cancer. [J]. Journal of Clinical Oncology .2012,17 (11 ): 988-993.

[8] Wang Libo, Xi Yan, Wang Yanrong, either morning both MMP-2 EGFR expression and MVD in non-small cell lung cancer [J] Chinese Journal of Clinical Oncology .2009,36 (22):... 1300-1303.

[9] Siegel RL, Miller KD, Jemal A. Cancer statistics, 2015 [J] CA Cancer J Clin, 2015, 65 (1): 5-29. 